\title{
APOLARITY OF TRILINEAR FORMS AND PENCILS OF BILINEAR FORMS*
}

\author{
R. M. THRALL
}

1. Introduction. In this note the idea of apolar trilinear forms, introduced earlier by the author, $\dagger$ is generalized, and the main theorems are given new proofs independent of the group theoretic methods and notation used then. The importance of the apolarity concept in trilinear form classification is illustrated by a numerical application. In $\S 3$ there is given a new method of classifying singular pencils of bilinear forms based on Dickson's minimal numbers.

2. Apolarity of trilinear forms. A trilinear form $F(x, y, z)$ $=\sum a_{h i j} x_{h} y_{i} z_{j}$, where $h$ runs from 1 to $l, i$ from 1 to $m$, and $j$ from 1 to $n$, has as two-way rank invariants $\S r_{h}, r_{i}, r_{j}$ the smallest numbers of variables $x, y, z$, respectively, in terms of which the form can be expressed. Let $F$ and $F^{\prime}=\sum b_{h i j} x_{h} y_{i} z_{j}$ be two trilinear forms in which the numbers of $x$ 's, $y$ 's, $z$ 's are $r_{h}, m, n$ and $r_{h}^{\prime}, m, n$, respectively, where $r_{h}$ and $r_{h}^{\prime}$ are the two-way $h$-rank invariants of $F$ and $F^{\prime}$.

DEFINITION. $F$ and $F^{\prime}$ are said to be $h$ apolar (relative to $\left.m, n\right) \|$ if

$$
\sum_{i, j=1}^{m, n} a_{h i j} b_{\nu i j}=0, \quad h=1, \cdots, r_{h} ; \nu=1, \cdots, r_{h}^{\prime},
$$

and

$$
r_{h}+r_{h}^{\prime}=m n .
$$

We define $i$ and $j$ apolarity analogously with respect to the $i$ and $j$ two-way rank invariants of $F$ and $F^{\prime}$. We shall apply the term apolar to two forms if they are $h, i$, or $j$ apolar. The following theorems concerning $h$ apolarity can, of course, be rephrased in terms of $i$ and $j$ apolarity.

We consider questions of existence and uniqueness properties of

* Presented to the Society, April 8, 1938.

$\dagger$ Metabelian groups and trilinear forms, American Journal of Mathematics, vol. 60 (1938), pp. 383-415.

$\ddagger$ Singular case of pairs of bilinear, quadratic, or Hermitian forms, Transactions of this Society, vol. 29 (1927), pp. 239-253.

$\S$ Cf. R. Oldenburger, On canonical binary trilinear forms, this Bulletin, vol. 38 (1932), p. 385.

$\|$ Evidently $m \geqq r_{i}, n \geqq r_{j}$. For most applications we take $m=r_{i}, n=r_{j}$. 
forms $F^{\prime}$ apolar to a given form $F$ (relative to $m, n$ ). Let $a_{h i j}=a_{h \lambda}$, where $\lambda=(i-1) n+j$. ${ }^{*}$ Defining $b_{\nu i j}=b_{\nu \lambda}$, we can write (1) as

$$
\sum_{\lambda=1}^{m n} a_{h \lambda} b_{\nu \lambda}=0, \quad h=1, \cdots, r_{h} ; \nu=1, \cdots, r_{h}=m n-r_{h} .
$$

The rank of $\left(a_{h \lambda}\right)$ is $r_{h}$, for if it were less, $F$ could be expressed in terms of less than $r_{h}$ variables $x$. The $b_{\nu \lambda}$ in $A^{\prime}$ can be interpreted as a set of $r_{h}^{\prime}$ linearly independent solutions of the equations $\sum_{\lambda=1}^{m n} a_{h \lambda} t_{\lambda}=0$, $\left(h=1, \cdots, r_{h}\right)$. It is well known that such solutions $b_{\nu \lambda}$ always exist, and that furthermore, if $c_{\mu \lambda}$ is a second set of solutions, constants $k_{\mu \nu}$ exist such that

$$
c_{\mu \lambda}=\sum_{\nu=1}^{r_{h^{\prime}}} k_{\mu \nu} b_{\nu \lambda}, \quad \mu=1, \cdots, r_{h}^{\prime},
$$

where $\left|k_{\mu \nu}\right| \neq 0 . \dagger$ Formula (1) is the usual definition of apolarity of two-way matrices. If we write $c_{\mu i j}=c_{\mu \lambda}$, $\left(3^{\prime}\right)$ becomes

$$
c_{\mu i j}=\sum_{\nu=1}^{r_{h^{\prime}}} k_{\mu \nu} b_{\nu i j}, \quad \mu=1, \cdots, r_{h}^{\prime} .
$$

If we subject the $x_{\nu}$ in $F^{\prime}$ to the non-singular linear transformation $x_{\nu}=\sum_{\mu} k_{\mu \nu} x_{\mu}^{\prime}, F^{\prime}$ becomes

$$
\sum_{\nu, i, j} b_{\nu i j}\left(\sum_{\mu} k_{\mu \nu} x_{\mu}^{\prime}\right) y_{i} z_{j}=\sum_{\mu, i, j}\left(\sum_{\nu} k_{\mu \nu} b_{\nu i j}\right) x_{\mu}^{\prime} y_{i} z_{j}=\sum_{\mu, i, j} c_{\mu i j} x_{\mu}^{\prime} y_{i} z_{j} .
$$

We have proved the following theorem:

THEOREM 1. For every trilinear form $F$ with invariants $r_{h}, r_{i}, r_{j}$ and every pair of integers $m\left(\geqq r_{i}\right), n\left(\geqq r_{j}\right)$ there exists a trilinear form $F^{\prime}$ which is $h$ apolar to $F$. Any second form $F^{\prime \prime}$ which is $h$ apolar to $F$ (relative to $m, n$ ) is conjugate to $F^{\prime}$ under non-singular linear transformations on the variables $x_{\nu}$ of $F^{\prime}$.

Since the steps are all rational, the coefficients of $F^{\prime}$ can be chosen from the field of the coefficients of $F$.

Two trilinear forms $F$ and $G$ are said to be equivalent, written $F \sim G$, if $F$ can be sent into $G$ by non-singular linear transformations on its sets of variables $x, y, z$, taken separately.

* The matrix $\left(a_{h \lambda}\right)$ is called the two-way h-display of $F$. See R. Oldenburger, Nonsingular multilinear forms and certain p-way matrix factorizations, Transactions of this Society, vol. 39 (1936), pp. 423-424.

† See, for example, J. H. M. Wedderburn, Lectures on Matrices, American Mathematical Society Colloquium Publications, vol. 17, New York, 1934, p. 19, Theorem 10. 
THEOREm 2. If $F \sim G$, and if $F^{\prime}, G^{\prime}$ are $h$ apolar to $F, G$, respectively, (relative to the same numbers $m, n$ ), then $F^{\prime} \sim G^{\prime}$.

If $F$ can be sent into $G$ by transformations on $x$ alone, then $F$ is, by Theorem $1, h$ apolar to $G^{\prime}$; whence $F^{\prime} \sim G^{\prime}$. Next suppose that $G=\sum c_{h \mu j} x_{h} y_{\mu}^{\prime} z_{j}$ is obtained from $F$ by the transformation $T$, $y_{i}=\sum k_{\mu i} y_{\mu}^{\prime} ;$ that is, $c_{h \mu j}=\sum_{i} k_{\mu i} a_{h i j}$. Then let $F^{*}=\sum d_{\nu \mu j} x_{\nu} y_{\mu}^{\prime} z_{j}$ be obtained from $F^{\prime}=\sum b_{\nu \rho j} x_{\nu} y_{\rho} z_{j}$ by applying the transformation $y_{\rho}=\sum \kappa_{\mu \rho} y_{\mu}^{\prime}$, contragredient to $T$, that is, $\sum \kappa_{\mu \rho} k_{\mu i}=\delta_{\rho i}$ (Kronecker's $\left.\delta_{i j}\right)$. We see that $F^{*}$ is $h$ apolar to $G$, for

$$
\begin{aligned}
\sum_{\mu, j} d_{\nu \mu j} c_{h \mu j} & =\sum_{i, \rho, j}\left(\sum_{\mu} \kappa_{\mu \rho} k_{\mu i}\right) b_{\nu \rho j} a_{h i j}=\sum_{i, \rho, j} \delta_{i \rho} b_{\nu \rho j} a_{h i j} \\
& =\sum_{i, j} b_{\nu i j} a_{h i j}=0
\end{aligned}
$$

since $F^{\prime}$ and $F$ are $h$ apolar. Then $F^{\prime} \sim F^{*} \sim G^{\prime}$ gives $F^{\prime} \sim G^{\prime}$, where $G^{\prime}$ is any form $h$ apolar to $G$. By symmetry the same result holds for linear transformations on $z_{j}$. The remainder of the argument is obvious and will be omitted.

Theorem 2 enables us to speak of apolar classes of forms $[F]$ and $\left[F^{\prime}\right]$. ( $[F]$ denotes the class of forms equivalent to $F$.) Theorems 1 and 2 , together with their analogs for $i$ and $j$ apolarity, can be applied to reduce the problem of classifying all trilinear forms to that of classifying trilinear forms in which no one of the rank invariants $r_{h}, r_{i}, r_{j}$ is greater than half the product of the other two. To prove this we note first that no one of the $r_{\nu}$ can exceed the product of the other two. $\dagger$ Then it is obvious that one of $r_{h}$ and $r_{h}^{\prime}=r_{i} r_{j}-r_{h}$ does not exceed $r_{i} r_{j} / 2$.

An example will serve to illuminate such application. Let $F$ have $r_{h}=27, r_{i}=10, r_{j}=3$. Then if we take $m=10, n=3,[F]$ is $h$ apolar to a unique class of forms $\left[F^{\prime}\right]$ with $r_{h}^{\prime}=3, r_{i}^{\prime} \leqq 10, r_{j}^{\prime} \leqq 3$. As $[F]$ runs through the classes of forms $(27,10,3)$, there will be classes $\left[F^{\prime}\right]$ for each value of $r_{j}^{\prime} \leqq 3$ and $r_{h}^{\prime} / r_{j}^{\prime}=3 / r_{j}^{\prime} \leqq r_{i}^{\prime} \leqq 3 r_{j}^{\prime}=r_{i}^{\prime} r_{j}^{\prime}$. Hence, given the classes of forms with $\left(r_{h}, r_{i}, r_{j}\right)=(3,9,3),(3,8,3), \cdots,(3,1,3)$, $(3,6,2),(3,5,2), \cdots,(3,2,2),(3,3,1)$, we can at once write down the classes of forms $(27,10,3)$.

But in the same way, the forms $(3,9,3), \cdots,(3,5,3)$ are $i$ apolar to forms with $r_{h} \leqq 3, r_{i} \leqq 4, r_{j} \leqq 3$; and the forms $(2,6,3),(2,5,3)$, $(2,4,3)$ are apolar to forms with the $r_{\nu}$ all $\leqq 3$. Hence the classification of forms $(27,10,3)$ with $810=3 \cdot 10 \cdot 27$ coefficients can be re-

$\dagger$ H. R. Brahana, Metabelian groups and trilinear forms, Duke Mathematical Journal, vol. 1 (1935), p. 191. 
duced by apolarity considerations to the solution of linear equations and to the classification of forms in which all the $r_{\nu}$ are less than or equal to three or $\left(r_{h}, r_{i}, r_{j}\right)=(3,4,3)$ and in which at most 36 coefficients appear.

3. Pencils of bilinear forms. Consider the trilinear form

$$
G=\sum_{\nu=1}^{R} R_{\nu}+\sum_{\lambda=1}^{S} S_{\lambda}+T,
$$

where

$$
\begin{gathered}
T=z_{1} \sum_{\alpha, \beta=1}^{n} t_{\alpha \beta} x_{\alpha} y_{\beta}+z_{2} \sum_{\alpha=1}^{n} x_{\alpha} y_{\alpha}, \quad R_{\nu}=\sum_{\alpha=1}^{m_{\nu}}\left(z_{1} x_{\nu \alpha-1}+z_{2} x_{\nu \alpha}\right) y_{\nu \alpha}, \\
S_{\lambda}=\sum_{\alpha=1}^{n_{\lambda}} X_{\lambda \alpha}\left(z_{1} Y_{\lambda \alpha-1}+z_{2} Y_{\lambda \alpha}\right),
\end{gathered}
$$

and all of the variables in the forms $R_{\nu}, S_{\lambda}, T$ are linearly independent in the field $K$ of $G$. We suppose also that $m_{\nu} \geqq m_{\nu+1}, n_{\lambda} \geqq n_{\lambda+1}$. The form $G$ has two-way rank invariants

$$
\begin{aligned}
r_{h}=\sum_{\nu=1}^{R}\left(m_{\nu}+1\right)+\sum_{\lambda=1}^{S} n_{\lambda}+n, \quad r_{i} & =\sum_{\nu=1}^{R} m_{\nu}+\sum_{\lambda=1}^{S}\left(n_{\lambda}+1\right)+n, \\
r_{j} & =2 .
\end{aligned}
$$

The form $\bar{G}=\sum_{\nu=1}^{\bar{R}} \bar{R}_{\nu}+\sum_{\lambda=1}^{\bar{S}} \bar{S}_{\lambda}+\bar{T}$ is $h$ apolar to $G$ (relative to $r_{i}$ and 2$), \dagger$ where

$$
\begin{aligned}
\bar{T} & =z_{1} \sum_{\alpha=1}^{n} x_{\alpha} y_{\alpha}-z_{2} \sum_{\alpha, \beta=1}^{n} t_{\beta \alpha} x_{\alpha} y_{\beta}, \\
\bar{R}_{\nu} & =\sum_{\alpha=1}^{m_{\nu}-1} x_{\nu \alpha}\left(z_{1} y_{\nu \alpha+1}-z_{2} y_{\nu \alpha}\right), \\
\bar{S}_{\lambda} & =\sum_{\alpha=1}^{n_{\lambda}+1} Y_{\lambda \alpha-1}\left(z_{1} X_{\lambda \alpha}-z_{2} X_{\lambda \alpha-1}\right),
\end{aligned}
$$

and the variables $x$ and $y$ are all independent. We have $\bar{S}=S, R-\bar{R}$ $=j_{1}$, where $j_{\alpha}$ is the number of $m_{\nu}$ equal to $\alpha$.

If in $G$ we let $z_{1}^{\prime}=-z_{2}, z_{2}^{\prime}=z_{1}, x_{\alpha}=y_{\alpha}^{\prime}, x_{\nu \alpha}=y_{\nu \alpha}^{\prime}, X_{\lambda \alpha}=Y_{\lambda \alpha}^{\prime}, y_{\alpha}=x_{\alpha}^{\prime}$, $y_{\nu \alpha}=x_{\nu \alpha-1}^{\prime}, Y_{\lambda \alpha}=X_{\lambda \alpha-1}$, we obtain a form $G^{\prime}$ identical in form with $G$, having $T^{\prime}(x, y) \equiv T(x, y)$ and the numbers $m_{\nu}^{\prime}=m_{\nu}-1, n_{\lambda}^{\prime}=n_{\lambda}+1$.

$\dagger$ That condition (1) for apolarity is satisfied is obvious if the two-way $h$-displays of $G$ and $\bar{G}$ are written down. For (2) we note that $\bar{r}_{h}+r_{h}=\left(\sum\left(m_{\nu}-1\right)+\sum\left(n_{\lambda}+2\right)\right.$ $+n)+\left(\sum\left(m_{\nu}+1\right)+\sum n_{\lambda}+n\right)=2\left(\sum m_{\nu}+\sum\left(n_{\lambda}+1\right)+n\right)=2 r_{i}$. 
(We omit $R_{\nu}^{\prime}$ if $m_{\nu}^{\prime}=0$.) Then $S^{\prime}=S, R^{\prime}=R-j_{1}$. Hence $G^{\prime}$ has twoway rank invariants

$$
\begin{aligned}
& r_{h}^{\prime}=\sum_{\nu=1}^{R^{\prime}} m_{\nu}+\sum_{\lambda=1}^{S}\left(n_{\lambda}+1\right)+n, \\
& r_{i}^{\prime}=\sum_{\nu=1}^{R^{\prime}}\left(m_{\nu}-1\right)+\sum_{\lambda=1}^{S}\left(n_{\lambda}+2\right)+n .
\end{aligned}
$$

Considered as a pencil of bilinear forms with parameter $z=\left(z_{1}, z_{2}\right)$, $G$ has minimal numbers $m_{\nu}$ and $n_{\lambda}$ and invariant factors (aside from 1) equal to those of $T$. Dickson* has proved that any pencil of bilinear forms $F$ can be put in the canonical form $G$ by non-singular (rational) transformations on the variables $x, y, z$. We have then $F \sim G$. Let $\bar{F}$ be $h$ apolar to $F$ (relative to $r_{i}$ and 2). Then, by Theorem $2, \bar{F} \sim \bar{G}$; and if $F^{\prime}$ is for $\bar{F}$ the analogue $\dagger$ of $G^{\prime}$ for $\bar{G}$, then $F^{\prime} \sim G^{\prime}$. This means in particular that $F^{\prime}$ has the same minimal numbers as $G^{\prime}$ and invariant factors projectively equivalent to those of $F$ (since $F, G, G^{\prime}$, $F^{\prime}$ have, in turn, projectively equivalent invariant factors).

Let $F^{(\alpha)}$ be derived from $F^{(\alpha-1)}$ as $F^{\prime}$ is derived from $F^{(0)}=F$. We propose to calculate the minimal numbers $m_{\nu}$ from the sequence of two-way rank invariants $r_{h}^{(\alpha)}, r_{i}^{(\alpha)}$. Since these invariants are ranks of two-way matrices, we will then have the $m_{\nu}$ expressed in terms of ranks of matrices, obtainable from $F$ by solving linear equations.

To effect this calculation we shall evaluate the $j_{\alpha}$ defined above. By hypothesis $m_{1}$ is the biggest of the $m_{\alpha}$, hence $j_{\alpha}=0$ for $\alpha>m_{1}$. Hence, as soon as we know the sequence $j_{\alpha},\left(\alpha=1, \cdots, m_{1}\right)$, we know all of the minimal numbers $m_{\nu}$. In particular $R^{(0)}=R=\sum_{\alpha=1}^{m_{1}} j_{\alpha}$, and $\sum_{\nu=1}^{R} m_{\nu}=\sum_{\alpha=1}^{m_{1}} \alpha j_{\alpha}$.

We saw above that $j_{1}=R^{(0)}-R^{(1)}$. Now

$$
\begin{aligned}
r_{h}{ }^{(0)}-r_{h}{ }^{(1)}= & \left(\sum_{\nu=1}^{R^{(0)}}\left(m_{\nu}+1\right)+\sum_{\lambda=1}^{S^{(0)}} n_{\lambda}+n\right) \\
& -\left(\sum_{\nu=1}^{R^{(0)}} m_{\nu}+\sum_{\lambda=1}^{S^{(0)}}\left(n_{\lambda}+1\right)+n\right) \\
= & {\left[R^{(0)}+\left(R^{(0)}-R^{(1)}\right)-S^{(0)},\right.}
\end{aligned}
$$

* Loc. cit., in particular Theorem 2, p. 248 and Theorem 3, p. 251. These results are stated for pairs of bilinear forms, but the numbers $m_{\nu}, n_{\lambda}$ and the projective invariants of the invariant factors will constitute a complete set of invariants for pencils of bilinear forms. We use here this generalization of Dickson's result.

$\dagger$ That is, the matrix of $F^{\prime}$ is the transpose of that of $F$. 
and similarly $r_{i}{ }^{(0)}-r_{i}{ }^{(1)}=R^{(0)}-S^{(0)}$. Hence $j_{1}=R^{(0)}-R^{(1)}=\left(r_{h}{ }^{(0)}\right.$ $\left.-r_{h}^{(1)}\right)-\left(r_{i}{ }^{(0)}-r_{i}{ }^{(1)}\right)=\left(r_{h}^{(0)}-r_{i}{ }^{(0)}\right)-\left(r_{h}^{(1)}-r_{i}{ }^{(1)}\right)$. By induction on $\alpha$ we show that $j_{\alpha}=\left(r_{h}^{(\alpha-1)}-r_{i}^{(\alpha-1)}\right)-\left(r_{h}^{(\alpha)}-r_{i}^{(\alpha)}\right)$, which is the desired result.

The analogous process when we use $i$ apolarity gives the numbers $n_{\lambda}$.

The following inequality gives an upper limit for $m_{1}$ and is of use in determining the number of steps the above procedure will require. If $M<m_{1}$, then $R>\sum_{\alpha=1}^{M} j_{\alpha}$ and

$$
\sum_{\alpha=1}^{M} \alpha j_{\alpha}+(M+1) \leqq \sum_{\alpha=1}^{M} \alpha j_{\alpha}+m_{1} \leqq \sum_{\alpha=1}^{m_{1}} \alpha j_{\alpha}=\sum_{\nu=1}^{R} m_{\nu}
$$

But

$\sum_{\nu=1}^{R} m_{\nu}=r_{h}-n-\sum_{\lambda=1}^{S} n_{\lambda}-R \leqq r_{h}-n-R-S \leqq 2 r_{h}-r_{i}-n-2 R$.

$\left(S=R+r_{i}-r_{h}.\right)$ Hence $M<2 r_{h}-r_{i}-n-\sum_{\alpha=1}^{M}(\alpha+2) j_{\alpha}-2$. Therefore $m_{1}$ is less than the smallest integer $M$ for which this inequality does not hold. Or, if one computes the rank, $r_{h}-R=r_{i}-S$, of the characteristic matrix $M_{z}(z)$ of $F$, the value of $R$ thus obtained, together with $R=\sum_{\alpha=1}^{m_{1}} j_{\alpha}$, shows one exactly when enough steps have been taken.

Dickson's process* for determining $m_{\nu}, n_{\lambda}$ requires $R+S$ steps, each of which involves solving several sets of linear equations, and finding the minimum degree $m_{R-\alpha}$ in $z$ of the forms $A_{h}^{(\alpha)}$ such that $\sum A_{h}{ }^{(\alpha)} F_{h}(\alpha) \equiv 0$ in $z$ and $y$, where $F_{h}^{(\alpha)}$ is the coefficient of $x_{h}$ in $F^{(\alpha)}$ (the form obtained by the $(\alpha-1)$ st step). No method, other than trial and error, is given for determining $m_{R-\alpha}$.

The apolarity process requires $m_{1}+n_{1}$ steps, each of which involves solving one set of linear equations and evaluating the rank of a matrix (which is also the first step in solving the next set of linear equations). This gives, at the $\alpha$ th step, a specific formula for the number of $m_{\nu}$ equal to $\alpha$ (which is the chief value of the method).

Hence, if $R+S$ is small, Dickson's method is the shorter, but if $m_{1}+n_{1}<R+S$, or if $R+S$ and $m_{1}+n_{1}$ are large, the apolarity method is shorter.

UNIVERSITY OF MICHIGAN

* Loc. cit. 